\title{
Rheological and physicochemical characterization of alpha- tocopherol loaded lipid nanoparticles in thermoresponsive gel for topical application
}

\author{
Lim Qian Ying and Misni Misran* \\ Department of Chemistry, Faculty of Science, University of Malaya, 50603 Kuala Lumpur, Malaysia \\ * Corresponding author: misni@um.edu.my
}

Article history

Received 18 February 2017

Accepted 4 July 2017

\begin{abstract}
Features of a thermoresponsive gel are advantageous for topical application. Lipid nanoparticles is incorporated as a carrier for a hydrophobic active ingredient which is not stable in the aqueous gel system. In this work, a thermoresponsive gel mixture consists of carboxymethyl cellulose (CMC) and iota-carrageenan (I-C) was prepared. Nanostructured lipid carriers (NLC) made of fatty acids and lecithin was incorporated into the gel formulation as a carrier for alpha-tocopherol. Temperature effect on the rheological behavior of the prepared gel mixture was investigated. The NLC was evaluated for its mean particle size, zeta potential, morphology, encapsulation efficiency and in vitro drug release. Physicochemical characterization showed that alpha-tocopherol loaded NLC being stored at $4^{\circ} \mathrm{C}$ was stable for 1 month and encapsulation efficiency of alpha-tocopherol was more than $90 \%$ for all formulations due to its hydrophobicity to stay in the lipid matrix. In vitro release studies proved that NLC was able to provide slow release of loaded alpha-tocopherol. On the other hand, the phase transition from gel-like to liquid-like of the formulated gel mixture was proven to be activated by temperature changes. The rheological gelling point of the gel mixture was represented by the crossover point of storage modulus, G' and loss modulus, $G$ " which was obtained in the vicinity of body temperature $37^{\circ} \mathrm{C}$. This property is useful in topical application for its better spreadability on skin upon usage and hence better penetration for the alpha-tocopherol loaded NLC across the skin barrier. Prolong release of alpha-tocopherol with enhanced stability is favored as alpha-tocopherol is commonly known for its potent antioxidant activities. These results suggested that gel mixture of $\mathrm{CMC}$ and $\mathrm{I}-\mathrm{C}$ is a good candidate to be developed as a thermoresponsive gel while lipid nanoparticles is a promising carrier system for alpha-tocopherol in topical use.
\end{abstract}

Keywords: Rheology, thermoresponsive gel, lipid nanoparticles, topical application

\section{INTRODUCTION}

Topical drug delivery represents a promising route mainly for treatment of localized skin diseases and cosmetic use. A gel is commonly defined as a phase dispersed in another continuous phase forming a three-dimensional network by chemical covalent bond or physical interactions (Nishinari, 2009). It is one of the most general semisolid formulations used in topical application for its good stability against separation and lack of greasiness upon usage. However, active compounds tend to degrade in the dispersing aqueous medium of the gel during storage, thus greatly reduces the effectiveness of the product. Moreover, active compounds are difficult to penetrate across our skin barrier. In fact, skin being the organ most exposed to the environment prevents most external substances penetrating into our body (Friberg, 1990). In order to enhance the penetration and stability of active compounds, a carrier system is incorporated into the gel formulation to provide a more efficient drug delivery.

Colloidal carrier systems had been widely employed to overcome the limitations of conventional dermal formulations in pharmaceutical and cosmetics industries. As an alternative to emulsions, liposomes and polymeric nanoparticles, lipid nanoparticles such as solid lipid nanoparticles (SLN) and nanostructured lipid carriers (NLC) were developed not only to provide improved features but also to meet the industrial needs including low cost and easiness for large scale production. Those features found in lipid nanoparticles are advantageous for dermal products for instances occlusive properties, modified release profile and avoidance of systemic uptake (Beck et al., 2011). Lipid nanoparticles are mainly utilized to load hydrophobic drug in its lipid core or lipid outer shell depending on the production method (Üner et al., 2007). The encapsulated drug is therefore protected against chemical degradation caused by the surrounding environment and dispersing medium such as light, oxidation and hydrolysis by water. In addition, the nanoparticles provide prolong release of drug to enhance its effectiveness and avoid irritation to the skin (Dingler et al., 1999; Grana et al., 2013).

Alpha-tocopherol is a form of lipid-soluble vitamin $\mathrm{E}$ with potent antioxidant properties essential for health. Oxidation is always related to numerous diseases especially cancer and aging, therefore daily intake of vitamin E is necessary (Podda et al., 2001; Rizvi et al., 2014). Besides dietary intake either from food or supplements, vitamin E can be applied topically to nourish our skin with the main purpose to prevent aging. Shylaja et al. (2016) had shown the enhanced stability and slow release property of vitamin E loaded in SLN.

In this study, alpha-tocopherol was loaded in lecithin-fatty acid nanoparticles which were then incorporated into a thermoresponsive gel mixture. The aim of employing a thermoresponsive gel instead of a 
conventional gel system is to enhance its spreadability upon usage by manipulating the transition temperature of gel from solid-like to liquidlike in the vicinity of body temperature. The physicochemical characteristics and release profile of alpha-tocopherol loaded NLC was investigated while the rheological behavior of the gel was evaluated.

\section{EXPERIMENTAL}

\section{Materials}

Fatty acids (stearic acid and oleic acid), Tween 80, iotacarrageenan and phosphate buffered saline tablets were purchased from Sigma Aldrich (St. Louis, USA). Calcium chloride anhydrous was obtained from Merck while carboxymethyl cellulose was bought from Dai-Ichi Kogyo Seiyaku (Kyoto, Japan), respectively. Lecithin was purchased from Alfa Aesar (Massachusetts, USA) and alphatocopherol was obtained from Spectrum (New Jersey, USA). All solutions and samples were prepared by using deionized water with resistivity of $18.2 \Omega \mathrm{cm}^{-1}$, which was supplied from a Barnstead Diamond Nanopure Water Purification unit coupled with a Barnstead Diamond RO unit (Barnstead International, USA).

\section{Preparation of nanostructured lipid carrier (NLC)}

NLC was prepared by hot homogenization method using a high shear homogenizer (Heidolph Silent Crusher M, Germany). Stearic acid, oleic acid and lecithin were heated at $80{ }^{\circ} \mathrm{C}$ until the mixture became clear liquid and alpha-tocopherol was added into the molten lipid. Surfactant solution (Tween 80) was pre-heated at the same temperature and dispersed into the lipid phase under strong agitation at $18,000 \mathrm{rpm}$ for 10 minutes. The hot emulsion was then poured into cold water at approximately $2^{\circ} \mathrm{C}$ under magnetic stirring where solidification of lipid occurred. The NLC dispersions were stored at $4{ }^{\circ} \mathrm{C}$ for further investigation.

\section{Preparation of thermoresponsive gel and NLC-gel}

Carboxymethyl cellulose (CMC) and iota-carrageenan $(l-\mathrm{C})$ were weighed and added into $0.06 \%(\mathrm{w} / \mathrm{w}) \mathrm{Ca}^{2+}$ solution. The mixture was then heated in a water bath up to $80^{\circ} \mathrm{C}$ until a clear gel was obtained and kept at room temperature for 24 hours before incorporation of NLC dispersion. $30 \%$ of NLC dispersion (w/w) was incorporated into the gel system and mixed homogeneously at $10,000 \mathrm{rpm}$ for 1 minute. The NLC-gel was stored at $4^{\circ} \mathrm{C}$ for 24 hours for further characterization.

\section{Mean particle size and zeta potential measurement of NLC}

The mean particle size and zeta potential of NLC were measured with Zetasizer Nano ZS (Malvern Instruments, U.K.) using Dynamic Light Scattering. NLC dispersions were diluted to an appropriate concentration before being placed into a $1 \mathrm{~cm}$ path length quartz cuvette for particle size measurement. A disposable folded capillary cell (DTS1070) was used to perform zeta potential measurement.

\section{Morphology of NLC}

The morphology of the alpha-tocopherol loaded NLC was observed by an Energy Filtered Transmission Electron Microscopy (TEM) model LIBRA 120 (Zeiss, Germany) with accelerating voltage of $120 \mathrm{kV}$. A drop of NLC was placed on a 400 mesh copper-coated carbon grid and stained with $2 \%$ phosphotungstic acid solution. The excess solution was removed by filter paper. After drying in a desiccator for 24 hours, the grid was ready to be examined by TEM.

\section{Encapsulation efficiency of alpha-tocopherol}

Encapsulation efficiency of alpha-tocopherol loaded NLC can be determined by utilizing centrifugal ultrafiltration method. The dispersion was filled into the upper chamber of a centrifugal filter tube with $50 \mathrm{kDa}$ molecular weight cut-off (Vivaspin 6, Sartorius Stedim Biotech, Germany) and centrifuged at 10,000 rpm for 1 hour. After centrifugation of the dispersion, the supernatant in bottom chamber was dissolved in methanol and vortex homogeneously. Alpha-tocopherol was detected spectrophotometrically (Cary 50 UV-Vis Spectrometer, Agilent Technologies, USA) at wavelength $290 \mathrm{~nm}$. The concentration of alpha-tocopherol in each sample was determined from a standard calibration curve. Encapsulation efficiency was calculated with the following equation:

$$
E E=\left(\frac{W_{T}-W_{F}}{W_{T}}\right) \times 100 \%
$$

where $E E$ is encapsulation efficiency of alpha-tocopherol in NLC, $W_{T}$ is the weight of alpha-tocopherol added during preparation and $W_{F}$ is the weight of unloaded alpha-tocopherol detected in filtered aqueous phase, respectively.

\section{In vitro release}

The release of alpha-tocopherol from alpha-tocopherol solution, alpha-tocopherol gel, alpha-tocopherol loaded NLC dispersion and alpha-tocopherol loaded NLC-gel at $37^{\circ} \mathrm{C}$ were investigated by using Automated Franz Diffusion Cell System (Microette Plus Autosampling System, Hanson Research Co., USA) with diffusate chamber volume of $4 \mathrm{~mL}$ and effective diffusion area of $0.636 \mathrm{~cm}^{2}$. The Franz diffusion cells were fitted with pre-hydrated regenerated cellulose membranes with $10 \mathrm{kDa}$ molecular weight cut-off. Ethanol: Phosphate Buffer Solution (PBS) $(76: 24 \mathrm{v} / \mathrm{v})$ was filled into the receptor chambers to ensure pseudo-sink conditions by increasing the solubility of the active compound in the receiving phase. Similar receptor medium had been used for release studies of vitamin E in previous studies (Sharipova et al., 2016; Shylaja et al., 2016; Yenilmez et al., 2011). The receiving phase was stirred continuously at $400 \mathrm{rpm}$ and thermostated at $37 \pm 1^{\circ} \mathrm{C}$. Approximately $1 \mathrm{~mL}$ of sample was loaded into each donor chamber and the experiments were run for 24 hours. Samples were withdrawn and replaced with fresh medium from the reservoir at each predetermined time interval. The amount of alpha-tocopherol can be determined by employing the spectrophotometric method (Cary 50 UVVis Spectrometer, Agilent Technologies, USA). The experiment was then repeated with alpha-tocopherol gel and alpha-tocopherol loaded NLC-gel at $30^{\circ} \mathrm{C}$.

\section{Rheological characterization of gel}

A strain rate/stress controlled Rheometer MCR 301 (Anton-Paar, Austria) was employed to carry out the rheological measurements of blank gel, NLC-gel and alpha-tocopherol loaded NLC-gel. All the tests were done at $25 \pm 1{ }^{\circ} \mathrm{C}$ (except temperature sweep) with measuring geometry cone and plate $1 \% 50 \mathrm{~mm}$ and a gap of $0.100 \mathrm{~mm}$. Viscometry test was first performed at controlled shear rate ranged from $0.01-100$ $\mathrm{s}^{-1}$. After that, oscillatory tests were started with amplitude sweep at controlled strain mode within strain range $1-1000 \%$ at constant frequency $1 \mathrm{~Hz}$ to obtain the linear viscoelastic region (LVR) for the following frequency and temperature sweep tests. Frequency sweep was then performed at constant strain $5 \%$ at frequency range $10-0.1$ Hz. Temperature sweep was carried out by heating the sample from $20^{\circ} \mathrm{C}$ to $80^{\circ} \mathrm{C}$ at a rate of $10^{\circ} \mathrm{Cmin}^{-1}$, at single frequency $1 \mathrm{~Hz}$ and constant strain $5 \%$. Solvent traps were used to minimize the evaporation of sample.

\section{RESULTS AND DISCUSSION}

\section{Mean particle size and zeta potential of NLC}

Amount of alpha-tocopherol added was found to affect the mean particle size of NLC and the stability of the formulation. The mean particle size and polydispersity index (PDI) were rising with the increasing amount of alpha-tocopherol (Fig. 1a). Sedimentation was observed in NLCs with $0.4 \%$ and $0.6 \%$ of alpha-tocopherol after 3 weeks, plausibly due to the denser particles formed during lipid solidification. The high amount of alpha-tocopherol, which possesses high viscosity, produced a highly viscous nanoemulsion hence forming dense nanoparticles upon solidification. Even though NLCs with $0.1 \%$ and $0.2 \%$ of alpha-tocopherol were having similar mean particle size after 1 month, $0.1 \%$ was considered more stable for its lower and steadier increment in mean particle size and PDI. The fluctuating mean particle size and PDI of NLCs with loaded alpha-tocopherol more than $0.1 \%$ was probably caused by the expulsion of alpha-tocopherol from the lipid matrix where the free alpha-tocopherol might form nanoemulsion with excess surfactant and consequently contributing to the higher PDI (Woo et al., 2014). 
On the other hand, the alpha-tocopherol loaded NLCs were having an insignificant difference of average zeta potential with the unloaded NLC and there are no much changes across the analysis time. This showed consistent condition on the surface structure of the particles. In addition, the zeta potential for all formulations was found lower than $30 \mathrm{mV}$ whereby it was generally accepted as a stable colloidal dispersion (Müller et al., 2001). Nevertheless, steric stabilizer present in the system was not taken into account in this prediction. For instance, Tween 80 as a nonionic surfactant could not ionize in aqueous system but it was able to adsorb onto the surface of particles, forming steric hindrance between the particles (Han et al., 2008).

(a)

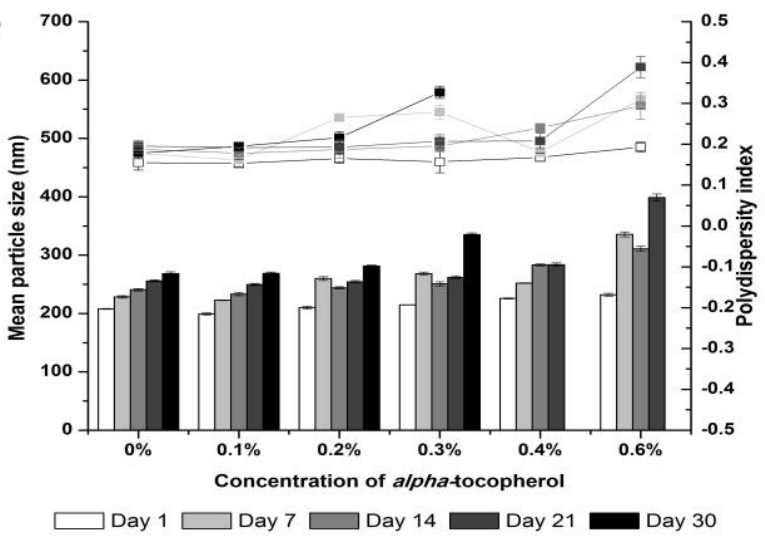

(b)

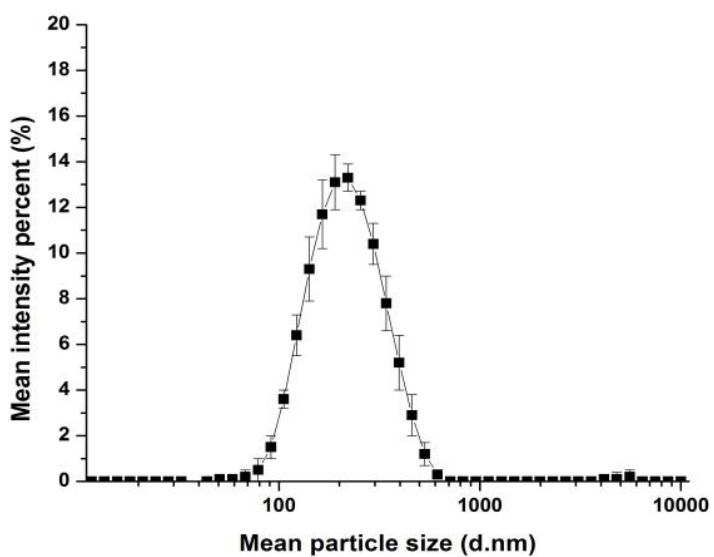

Fig. 1 (a) Mean particle size and PDI of NLCs as a function of concentration of alpha-tocopherol; (b) Mean intensity percent of $0.1 \%$ alpha-tocopherol loaded NLC as a function of mean particle size.

\section{Morphology of NLC}

TEM analyses of the alpha-tocopherol loaded NLCs revealed spherical shape which is in accordance with the results of particle size measurement. As shown in Fig. 2(a), NLC with $0.1 \%$ alpha-tocopherol had a monodisperse particle size and no evident aggregation. Nevertheless, NLC with a high amount of alpha-tocopherol showed potent aggregation that explains the sedimentation occurred after storage for 3 weeks (Fig. 2(b)). (a)

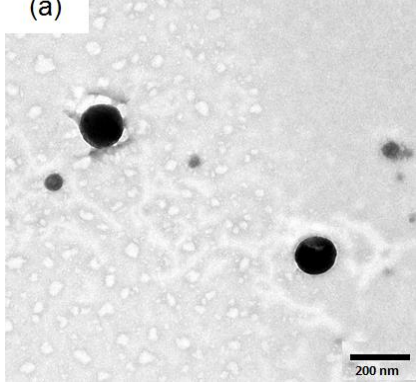

(b)

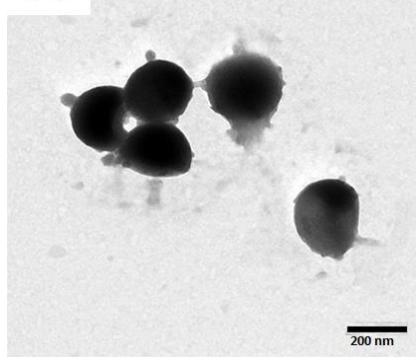

Fig. 2 TEM micrographs of NLCs loaded with (a) $0.1 \%$ and (b) $0.6 \%$ alpha-tocopherol.

\section{Encapsulation efficiency of alpha-tocopherol}

Only stable formulations with no sedimentation and acceptable increment in particle size were evaluated the encapsulation efficiency of alpha-tocopherol. Encapsulation efficiency of alpha-tocopherol was high, more than $90 \%$ for all formulations under investigation. This observation might be attributed to its hydrophobicity to stay in the lipid matrix rather than the aqueous dispersing medium. In addition, incorporation of oleic acid into the solid lipid perturbed the crystalline matrix of the solid lipid matrix and hence increasing the loading space for active compound (Woo et al., 20.14). Therefore, the main criteria for selecting the optimum formulation were not based on the encapsulation efficiency but the stability of the formulation during storage.

\section{In vitro release}

NLC with $0.1 \%$ alpha-tocopherol was chosen as the optimized formulation to undergo the release studies at $37^{\circ} \mathrm{C}$. The release of alpha-tocopherol solution, alpha-tocopherol gel and alpha-tocopherol loaded NLC-gel were investigated as comparisons. With respect to the thermoresponsive behavior of the gel system, release profiles of the gel samples were also studied at $30^{\circ} \mathrm{C}$ as well. The release of alphatocopherol was supposed only from the lipid phase of NLC due to its poor solubility in the aqueous phase (Jenning et al., 2000). The cumulative alpha-tocopherol released from the samples at $37^{\circ} \mathrm{C}$ (Fig. 3(a)) and $30^{\circ} \mathrm{C}$ (Fig. 3(b)) were plotted against time, respectively.

(a)

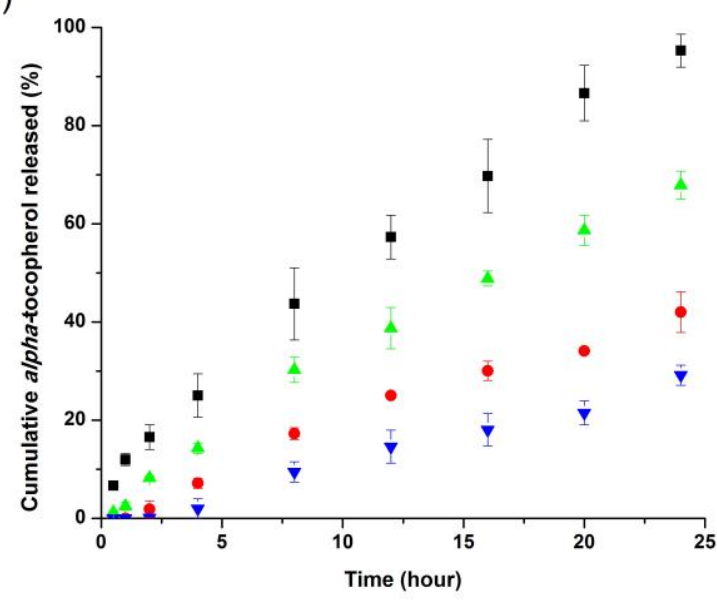

(b)

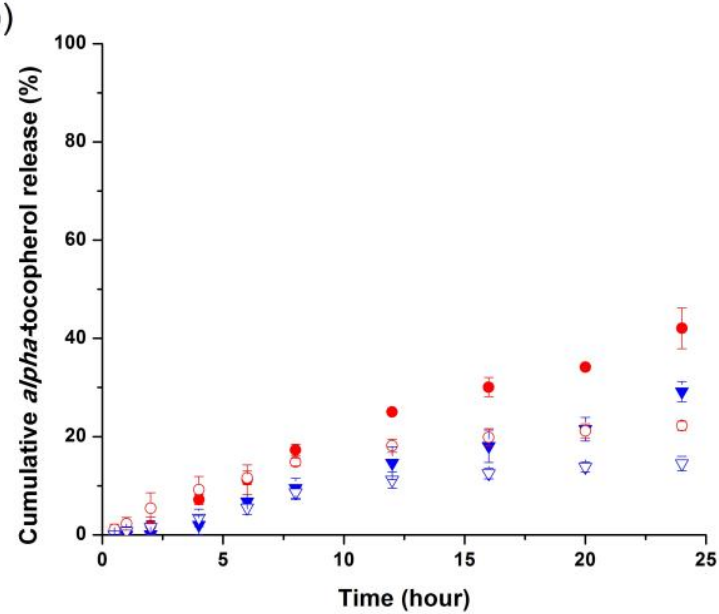

Fig. 3 In vitro release of (a) alpha-tocopherol solution (๘), alphatocopherol gel $(\bullet)$, alpha-tocopherol loaded NLC dispersion $(\boldsymbol{\Delta})$ and alpha-tocopherol loaded NLC-gel $(\boldsymbol{\nabla})$ over a period of 24 hours at $37^{\circ} \mathrm{C}$; (b) alpha-tocopherol gel $(\bullet)$ and alpha-tocopherol loaded NLC-gel $(\boldsymbol{\nabla})$ at $37^{\circ} \mathrm{C}$ (solid symbol $\bullet$ ) and $30^{\circ} \mathrm{C}$ (open symbol $\circ$ ).

As shown in Fig. 3(a), the release pattern of alpha-tocopherol from solution and NLC dispersion were similar but NLC dispersion was 
proven its slow release property. The mobility of the active ingredient was limited by solid lipid matrix of NLC, compared with free motion in solution. Surprisingly, the alpha-tocopherol gel was released even slower than the alpha-tocopherol loaded NLC dispersion, indicating that the alpha-tocopherol was easier to diffuse through the lipid matrix compared with the aqueous gel matrix. The fast diffusion in NLC might be attributed to the fact that alpha-tocopherol enriched the outer shell of the particles instead of the lipid core (Fig. 4). This is in agreement with the previous study where the shell enrichment model was proposed for hot homogenization production method (Üner et al., 2007). In addition, the gel samples had higher viscosity, making the active compound more difficult to diffuse. As expected, the alpha-tocopherol loaded NLC-gel gave the lowest cumulative release after 24 hours since the active compound had to diffuse through both the lipid matrix and gel matrix before entering the receiving phase.

On the other side, cumulative release of alpha-tocopherol from the gel samples at $30^{\circ} \mathrm{C}$ was significantly lower than that at $37^{\circ} \mathrm{C}$ (Fig. $3(\mathrm{~b}))$. At $30^{\circ} \mathrm{C}$, the gel samples in the donor chamber behave like solid with rigid gel network. The high viscosity increased the difficulty for the active ingredients to diffuse through the gel matrix. In contrast, the liquid-like property became dominant in the gel samples at $37^{\circ} \mathrm{C}$, leading to faster diffusion of alpha-tocopherol. This observation
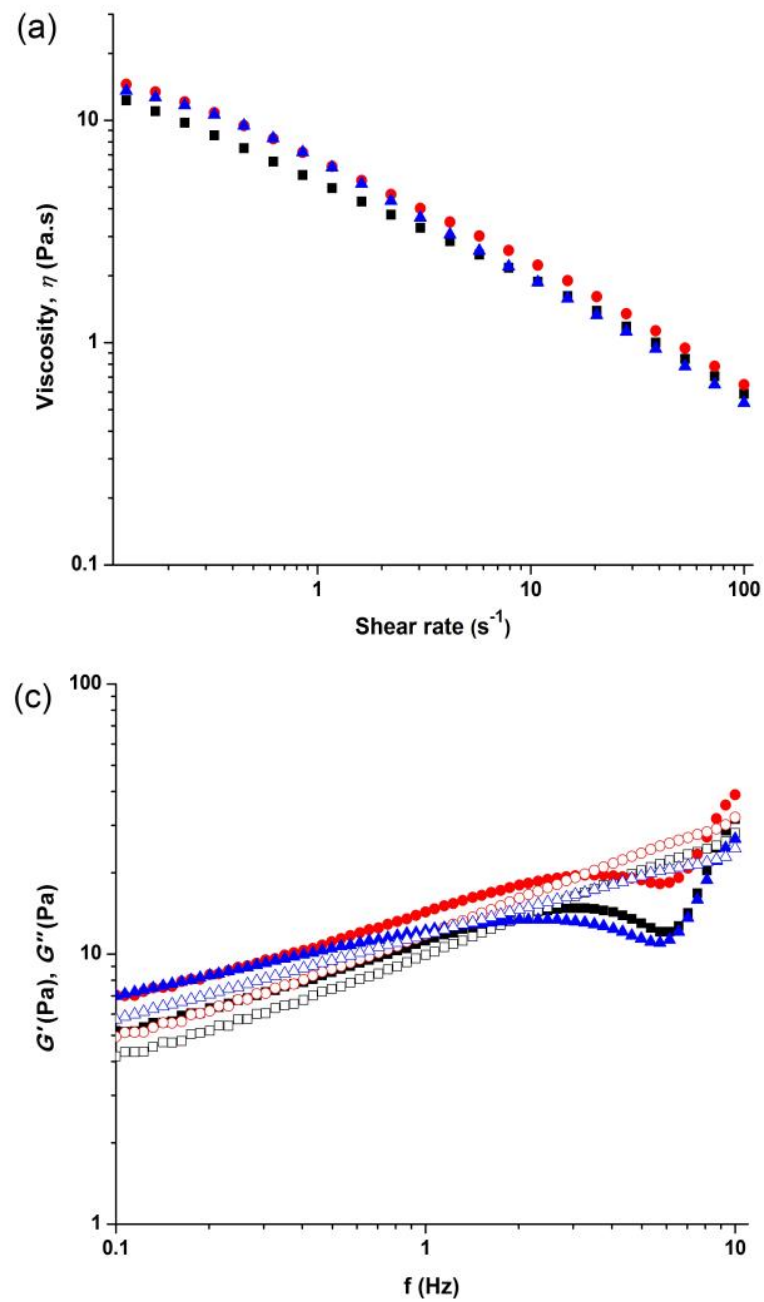

corresponds with the hypothesis made earlier that the optimized thermoresponsive gel system posesses a better flowing profile and hence better release of the active ingredients at body temperature.

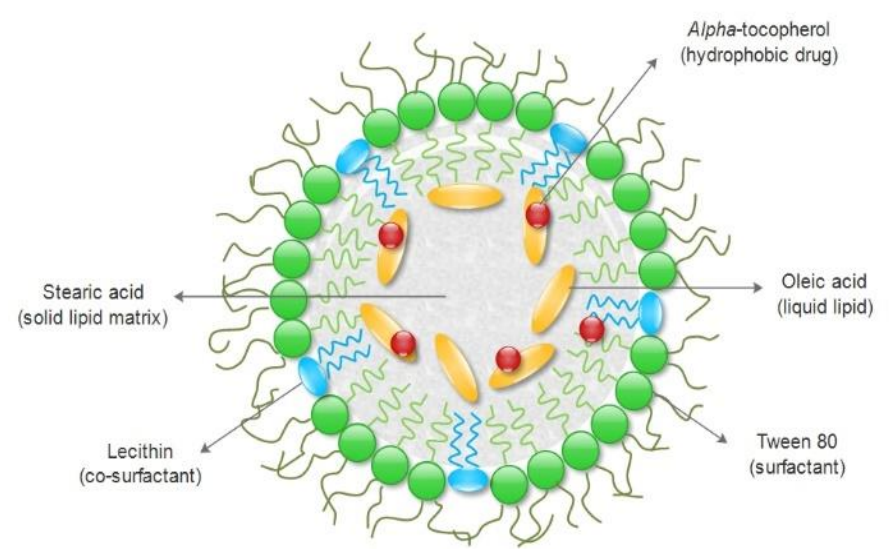

Fig. 4 Schematic illustration of alpha-tocopherol NLC.
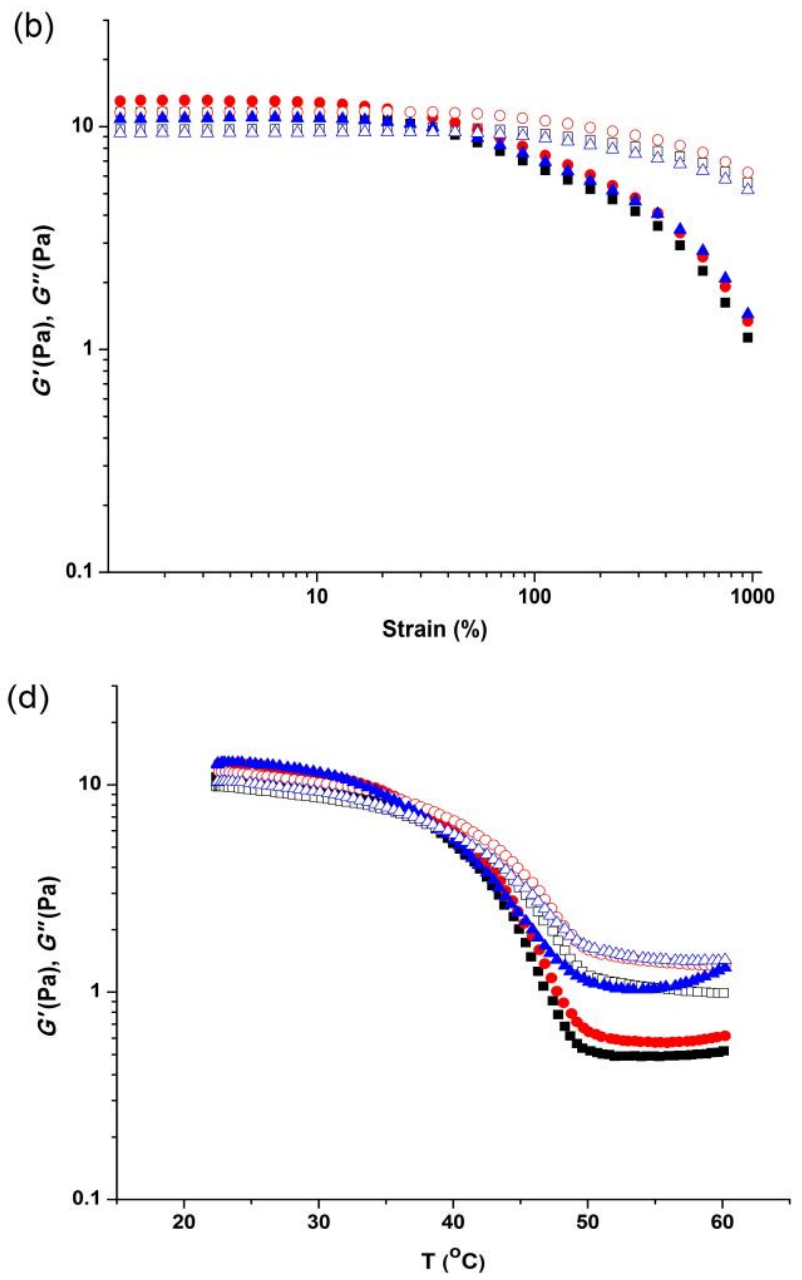

Fig. 5 Rheological measurement of sample blank gel $(\boldsymbol{\bullet})$, NLC-gel $(\bullet)$ and alpha-tocopherol loaded NLC-gel $(\boldsymbol{\Lambda})$ : (a) $\eta$ as a function of shear rate in viscometry; G' (solid symbol $\mathbf{\square}$ ) and G" (open symbol $\square$ ) (a) as a function of strain in amplitude sweep; (b) as a function of frequency in frequency sweep and (d) as a function of temperature in temperature sweep.

\section{Rheological characterization of gel}

Understanding the rheological properties is vital to reveal the microstructural environment and mobility, hence deduce the drug diffusion and compatibility. From Fig. 5(a), the flowing curves of the gel samples displayed shear thinning behavior where the viscosity, $\eta$ decreased with increasing shear rate. This non-Newtonian behavior is desired to provide slow flow from the bottle and easy distribution on the skin (Islam et al., 2004). At higher shear rates, the disentanglement of polymer occurred due to the deformation applied, consequently the active compound entrapped was able to diffuse following the direction of flow.

The oscillation tests were initiated with amplitude sweep to obtain the linear viscoelastic region (LVR) of the samples which indicates the maximum strain capable by the structure before it collapsed. All the samples exhibited solid-like properties at lower strain but viscous components dominate when strain applied reached approximately $30 \%$ 
(Fig. 5(b)). Therefore, a strain value of $5 \%$ was chosen for the subsequent frequency and temperature sweep tests to ensure the gel structure was not destroyed by the strain applied.

In frequency sweep (Fig. 5(c)), storage modulus, $G$ ' were found predominant at the lower frequency, showing the good stability of the gel at long time scale, i.e. during storage. However, a weak structure was characterized by high loss modulus, $G$ "' over $G$ ' at the higher frequency. This represents its good spreadability at short time scale, i.e. application on the skin. Furthermore, the rheological gelling point determined by the crossover point of $G^{\prime}$ and $G^{\prime \prime}$ happened at a temperature between $35^{\circ} \mathrm{C}-40^{\circ} \mathrm{C}$ for all the samples (Fig. $5(\mathrm{~d})$ ). This phase transition from solid-like to liquid-like at a temperature in the vicinity of body temperature $37^{\circ} \mathrm{C}$ again contributes to better spreadability of the gel upon application on the skin.

Basically, the rheological measurements indicated that all the gel samples behave similarly. Nevertheless, the NLC-gel had slightly higher $\eta$ and $G$ ' compared with the blank gel, owing to the presence of lipid nanoparticles. This suggested that NLC was able to store the applied energy in their elastic components under shear stress applied. The interactions between the nanoparticles and the polymer chains of the gel might also lead to the increased elastic behavior. It was proposed that hydrogen bonds were formed between the oxygen atoms in the fatty acid and the hydroxyl groups $(\mathrm{O}-\mathrm{H})$ at the backbone of the polymer chains in the gel matrix (Chieng et al., 2010; Tan et al., 2014). Despite that, the difference between alpha-tocopherol loaded NLC-gel and nonloaded NLC-gel was negligible. This revealed that the alphatocopherol was encapsulated within the lipid matrix, without disturbing the gel network.

\section{CONCLUSION}

In the present study, NLC made of stearic acid as solid lipid, oleic acid as liquid lipid, Tween 80 and lecithin as surfactants, was prepared by employing hot homogenization method. Since the encapsulation efficiency of alpha-tocopherol in the NLC was high for all formulations, the optimized formulation was determined from the stability profile according to the mean particle size and PDI measurements. $0.1 \%$ alpha-tocopherol loaded NLC was then incorporated into a thermoresponsive gel consists of $l$-C and CMC. As expected, NLC released the active compound at a slower rate compared with the free alpha-tocopherol solution. Incorporation of alphatocopherol loaded NLC into the gel matrix even delayed the release rate. Rheological measurements indicated that the gel was beneficial in topical use for its shear thinning behavior under shear stress applied. The thermally activated phase transition from gel-like to liquid-like at a temperature close to the body temperature could improve spreadability of the gel and hence provide better delivery of the active compound, in line with the release profile of the gel samples at $30^{\circ} \mathrm{C}$ and $37^{\circ} \mathrm{C}$. At the meanwhile, the rigidity of the gel was more pronounced with the incorporation of NLC, suggesting enhanced stability of NLC in the gel system. Considering the results obtained in the study, the thermoresponsive gel mixture of $l$-C and CMC is likely to be developed as a topical formulation while lipid nanoparticles is a promising carrier system for alpha-tocopherol.

\section{ACKNOWLEDGEMENT}

The authors would like to thank the University of Malaya and the Ministry of Higher Education Malaysia for financially supporting the work (PPP Grant no. PG114-2016A, UMRG Grant no. RP022C-16SUS and FRGS Grant no. FP013-2015A).

\section{REFERENCES}

Guimarães, K. L., Inês Ré, M. 2011. Lipid nanoparticles as carriers for cosmetic ingredients: The first (SLN) and the second generation (NLC). In Beck, R., Guterres, S., Pohlmann, A. (Eds.). Nanocosmetics and Nanomedicines: New approaches for skin care. (pp.101-122) Berlin Heidelberg: SpringerVerlag.

Chieng, Y. Y., Chen, S. B. 2010. Rheological study of hydrophobically modified hydroxyethyl cellulose and phospholipid vesicles. Journal of Colloid and Interface Science 349, 236-245.

Dingler, A., Blum, R. P., Niehus, H., Muller, R. H., Gohla, S. 1999. Solid lipid nanoparticles (SLNTM/LipopearlsTM) a pharmaceutical and cosmetic carrier for the application of Vitamin E in dermal products. Journal of Microencapsulation 16, 6, 751-767.

Friberg, S. E. 1990. Micelles, microemulsions, liquid crystals and the structure of stratum corneum lipids. Journal of the Society of Cosmetic Chemists 41 , $3,155-171$.

Grana, A., Limpach, A., Chauhan, H. 2013. Formulation considerations and applications of solid lipid nanoparticles. Retrieved from American Pharmaceutical Review website: http://www.americanpharmaceutical review.com/Featured-Articles/131176- Formulation- Considerations- andApplications-of-Solid-Lipid-Nanoparticles/

Han, F., Li, S., Yin, R., Liu, H., Xu, L. 2008. Effect of surfactants on the formation and characterization of a new type of colloidal drug delivery system: Nanostructured lipid carriers. Colloids and Surfaces A: Physicochemical and Engineering Aspects 315, 1-3, 210-216.

Islam, M. T., Rodriguez-Hornedo, N., Ciotti, S., Ackermann, C. 2004. Rheological characterization of topical carbomer gels neutralized to different pH. Pharmaceutical Research 21, 7, 1192-1199.

Jenning, V., Schäfer-Korting, M., Gohla, S. 2000. Vitamin A-loaded solid lipid nanoparticles for topical use: drug release properties. Journal of Controlled Release 66, 2-3, 115-126.

Müller, R. H., Jacobs, C., Kayser, O. 2001. Nanosuspensions as particulate drug formulations in therapy: Rationale for development and what we can expect for the future. Advanced Drug Delivery Reviews 47, 1, 3-19.

Nishinari, K. 2009. Some thoughts on the definition of a gel. In Tokita, M. and Nishinari, K. (Eds.). Gels: Structures, Properties, and Functions: Fundamentals and Applications, pp. 87-94. Berlin, Heidelberg: Springer.

Podda, M., Grundmann-Kollmann, M. 2001. Low molecular weight antioxidants and their role in skin ageing. Clinical and Experimental Dermatology 26, 7, 578-582.

Rizvi, S., Raza, S. T., Ahmed, F., Ahmad, A., Abbas, S., Mahdi, F. 2014. The role of Vitamin E in human health and some diseases. Sultan Qaboos University Medical Journal 14, 2, e157-e165.

Sharipova, A. A., Aidarova, S. B., Grigoriev, D., Mutalieva, B., Madibekova, G., Tleuova, A., Miller, R. 2016. Polymer-surfactant complexes for microencapsulation of vitamin $\mathrm{E}$ and its release. Journal $f$ Colloids and Surfaces B: Biointerfaces 137, 1, 152-157.

Shylaja, P. A., Mathew, M. M. 2016. Preparation and characterization of alpha tocopherol loaded solid lipid nanoparticles by hot homogenization method. International Journal of Pharmacy \& Pharmaceutical Research. Human Journals 7, 1, 437-448.

Tan, H. W., Misran, M. 2014. Effect of chitosan-modified fatty acid liposomes on the rheological properties of the carbohydrate-based gel. Applied Rheology 24, 34839.

Üner, M., Yener, G. 2007. Importance of solid lipid nanoparticles (SLN) in various administration routes and future perspectives. International Journal of Nanomedicine 2, 3, 289-300.

Woo, J. O., Misran, M., Lee, P. F., Tan, L. P. 2014. Development of a controlled release of salicylic acid loaded stearic acid-oleic acid nanoparticles in cream for topical delivery. The Scientific World Journal 2014, 1-7. http://dx.doi.org/10.1155/2014/205703

Yenilmez, E., Baasaran, E., Yazan, Y. 2011. Release characteristics of vitamin E incorporated chitosan microspheres and in vitro-in vivo evaluation for topical application. Carbohydrate Polymers 84, 2, 807-811. 